\title{
Review of Atypical Organometallic Compounds as Antimalarial Drugs
}

\author{
Samuel K. Kwofie, ${ }^{1,2,3}$ Emmanuel Broni (D), ${ }^{1}$ Bismark Dankwa (D), ${ }^{4}$ Kweku S. Enninful $\left(\mathbb{D},{ }^{4}\right.$ \\ Joshua Teye, ${ }^{1}$ Cedar R. Davidson, ${ }^{5}$ Josephine B. Nimely, ${ }^{5}$ J. Chioma Orizu, ${ }^{6}$ \\ Prakasha Kempaiah $\left(\mathbb{D},{ }^{3}\right.$ Brijesh Rathi $\mathbb{C}^{3,}, 7$ and Whelton A. Miller III $\oplus^{1,3,8}$ \\ ${ }^{1}$ Department of Biomedical Engineering, School of Engineering Sciences, College of Basic and Applied Sciences, \\ University of Ghana, PMB LG 77, Legon, Accra, Ghana \\ ${ }^{2}$ West African Center for Cell Biology of Infectious Pathogens, Department of Biochemistry, Cell and Molecular Biology, \\ College of Basic and Applied Sciences, University of Ghana, Legon, Accra, Ghana \\ ${ }^{3}$ Department of Medicine, Loyola University Chicago, Loyola University Medical Center, Maywood, IL 60153, USA \\ ${ }^{4}$ Department of Parasitology, Noguchi Memorial Institute for Medical Research (NMIMR), College of Health Sciences (CHS), \\ University of Ghana, Legon, Accra, Ghana \\ ${ }^{5}$ Department of Biology, Lincoln University, Chester County, PA 19352, USA \\ ${ }^{6}$ Department of Chemistry, Physics \& Engineering, Lincoln University, Chester County, PA 19352, USA \\ ${ }^{7}$ Laboratory for Translational Chemistry and Drug Discovery, Department of Chemistry, Hansraj College University Enclave, \\ University of Delhi, Delhi 110007, India \\ ${ }^{8}$ Department of Chemical and Biomolecular Engineering, School of Engineering and Applied Science, University of Pennsylvania, \\ Philadelphia, PA 19104, USA
}

Correspondence should be addressed to Whelton A. Miller III; wmiller6@luc.edu

Received 5 August 2019; Revised 16 January 2020; Accepted 10 February 2020; Published 20 May 2020

Academic Editor: Murat Senturk

Copyright ( 2020 Samuel K. Kwofie et al. This is an open access article distributed under the Creative Commons Attribution License, which permits unrestricted use, distribution, and reproduction in any medium, provided the original work is properly cited.

\begin{abstract}
Organometallic compounds are molecules that contain at least one metal-carbon bond. Due to resistance of the Plasmodium parasite to traditional organic antimalarials, the use of organometallic compounds has become widely adopted in antimalarial drug discovery. Ferroquine, which was developed due to the emergence of chloroquine resistance, is currently the most advanced organometallic antimalarial drug and has paved the way for the development of new organometallic antimalarials. In this review, a general overview of organometallic antimalarial compounds and their antimalarial activity in comparison to purely organic antimalarials are presented. Furthermore, recent developments in the field are discussed, and future applications of this emerging class of therapeutics in antimalarial drug discovery are suggested.
\end{abstract}

\section{Background: Organometallic Antimalarial Compounds and Their Derivatives}

Antimicrobial and antiparasitic resistance constitute a current threat facing the medical field today. Drug-resistant parasites force the development of new antiparasitic agents and depend on the interaction between functional groups in the drug and the target enzyme in the parasite for targeted therapy $[1,2]$. Current research is being placed into repurposing existing antibiotics, but, for the most diversity, new compounds need to be synthesized. Organometallic compounds are molecules that contain at least one metalcarbon bond. By introducing an organometallic moiety, there are greater possibilities and properties of the compound to work with and to be used in antimalarial agents [3]. Antimicrobial peptides (AMPs) modified with 
organometallic agents result in new organometallic AMPs that the parasite has never encountered and has not developed resistance to [4]. Quinine, chloroquine, hydroxychloroquine, mefloquine, primaquine, proguanil, doxycycline, sulfadoxine, pyrimethamine, artemether, lumefantrine, artesunate, and amodiaquine are some organic compounds which have been used in combination as antimalarial drugs. Chloroquine (Figure 1) was widely used due to its safety and low cost but the parasitic resistance to this drug has caused an obstacle to its treatment efficiency. This has caused an emerging need to develop new antimalarial drugs [5]. One way to develop new antimalarial drugs is to use the known organic structure of an antimalarial drug and to modify the unwanted properties using an organometallic moiety [3].

\section{Current Successes of Organometallic Antimalarial Drug Candidates in Medicine (Reported Cases)}

Chloroquine (CQ) and derivatives, e.g., hydroxychloroquine (HCQ) (Figure 2), some of the oldest antimalarial drugs, have become a starting point in the production of other antimalarial drugs such as mefloquine and quinine. Chloroquine's mechanism of action, in its uncharged form, diffuses freely into the digestive vacuole of the erythrocyte and is subsequently protonated. It then binds to the product of hemoglobin proteolysis, hematin [6,7]. Resistance to chloroquine may be associated with an increased level of drug efflux. Chloroquine-resistant strains have been reported to release chloroquine at a faster rate when compared to parasites sensitive to the drug [7]. Resistance to chloroquine is now widespread, and the loss of this drug has been a major setback for the effective treatment and control of malaria. There have been studies on rhodium and ruthenium combinations with chloroquine [5]. They show increased antimalarial activity against different strains of Plasmodium, especially against the CQ-susceptible strains. This led to the beginning of organometallic compounds as antimalarial agents. The poor efficacy of these derivatives pushed for a new drug discovery as an antimalarial agent. Ferrocene $(\mathrm{Fc})$ is an organometallic compound formed from two cyclopentadienyl ions combined with an iron $\left(\mathrm{Fe}^{2+}\right)$. Ferrocene's (Figure 3) sandwich-like structure is the metallocene (contains a transition metal and two cyclopentadienyl ligands coordinated). It is a promising choice because it is small and can permeate cellular membranes. It is also stable in an aqueous environment which allows versatility when it comes to its derivatives. Due to Fc exhibiting electrochemical behavior, it is a good candidate for drug design. Its lipophilic nature provides a favorable medium for the use of new biological applications such as anticancer and antimalarial drugs (ferrocifen, ferrocene, and ferroquine) [3].

Ferroquine (FQ) (Figure 4) is the first organometallic antimalarial drug. It contains a ferrocenyl group covalently flanked by a 4 -aminoquinoline and a basic alkylamine. Ferroquine is a derivative of $\mathrm{CQ}$ and ferrocene. Ferroquine envisaged structure was created to act similarly to chloroquine by localizing in the food vacuole of the parasite to

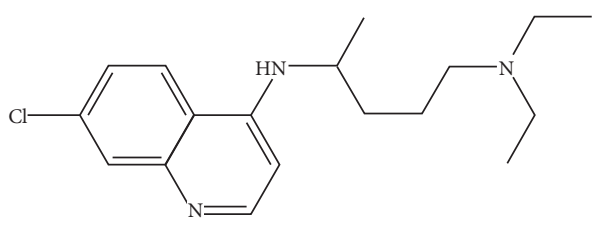

Figure 1: Chloroquine, CQ.

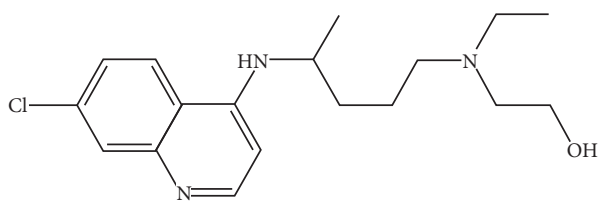

FIgURE 2: Hydroxychloroquine, HCQ.

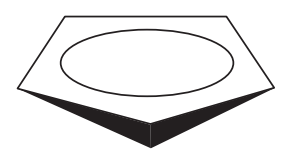

$\mathrm{Fe}$

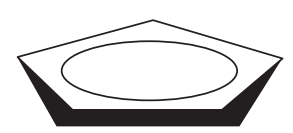

FIgURE 3: Ferrocene.

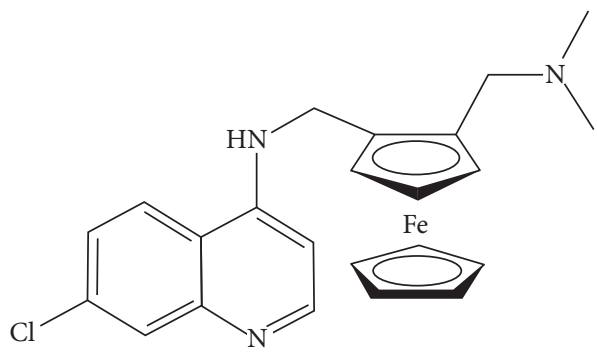

Figure 4: Ferroquine. $\mathrm{R}_{1}, \mathrm{R}_{2}=\mathrm{CH}_{3}$.

inhibit the formation of hemozoin [8]. Therefore, it was expected to act similarly to have identical mechanism to chloroquine when treating malaria. FQ is a good candidate for treating malaria because the parasite has not yet had the chance to develop a resistance to this antimalarial drug. A similar design strategy to that used for FQ was used on the side chains of quinine and mefloquine to replace the ferrocene moiety. Several ferrocenophane analogs of ferroquine derivatives are known to exhibit improved activity against certain strains of the Plasmodium parasite compared to their parent compound. The ruthenium-based analogs of FQ called ruthenoquine has been seen to be more active than ferroquine against chloroquine-resistant strains of Plasmodium parasite [9].

A study was conducted by Pierrot et al. on rats to determine the efficiency of FQ compared to the CQ. It was observed that after infection, although both treatments eventually showed no parasitemia, the rats treated with $\mathrm{FQ}$ were cured faster compared to those treated with its parent 
drug [10]. Another study done by Biot et al. tested FQ on various rodent malaria strains such as $P$. berghei, $P$. yoelii, and $P$. vinckei to determine efficient dosage. The curative test demonstrated that $P$. berghei and $P$. vinckei infections were cured with a dose of $8.3 \mathrm{mg} / \mathrm{kg} / \mathrm{d}$ of FQ for four days when CQ took 30 to $55 \mathrm{mg} / \mathrm{kg} / \mathrm{d}$. It also showed that FQ could be administered orally which is significant in the digestion of the drug [11]. To understand the pharmacokinetic and pharmacodynamic parameters of this compound, a study was performed by McCarthy et al. Eight volunteers aged 18-50 years were injected with $\sim 1800$ viable Plasmodium falciparum 3D7A-infected human erythrocytes and treated with one dose of $800 \mathrm{mg}$ ferroquine. The subjects were monitored throughout the study. It was determined that the ferroquine was absorbed quickly and the parasite reduction ratio was found to be 162.9 correlating to a clearance half-life of $6.5 \mathrm{~h}$. Ferroquine did show antiparasitic activity in all eight subjects and proved to be a good candidate moving forward with other clinical trials [12].

Ferrocene works synergistically when combined with known antimalarial drugs such as artemisinin, atovaquone, quine, and primaquine to produce different analogs and conjugates which are effective against the resistant and sensitive strains of the parasite. Although, these analogs were active against $P f 3 D 7 A$ strains, they were not as effective as ferroquine. Unlike chloroquine, chloroquine-resistant and chloroquine-susceptible strains of the Plasmodium parasites find it difficult to resist ferroquine due to its unique ferrocene side chain while retaining the aminoquinoline qualities of CQ.

\section{Antimalarial Activity of Organometallic Analogs of Known Antimalarial Drugs}

Ferroquine was discovered to have a great outcome in both CQ-resistant and CQ-sensitive P. falciparum because of its ability not only to inhibit the hemozoin formation, but also to demonstrate no immunotoxic effects in rat models [13]. FQ antimalarial activity came from the interaction of hydrogen in its lateral side chain which helps keep its geometric shape in place. Researchers further analyzed that the isosterism part of FQ had no interaction with the receptor but aided in the overall orientation and functionalities of the molecule. Additionally, FQ acts as a toxic-heme crystallization preventer by gathering in the digestive vacuoles of the parasite, causing damage to the hemozoin membrane, which results in the death of the parasite [13]. Furthermore, FQ generates a considerable amount of hydroxyl radicals in the digestive vacuoles of the parasite, causing the parasite to die [14]. When FQ is in the digestive vacuole of the malarial parasite, it undergoes a reversible one-electron redox reaction [14], resulting in the formation of salt and hydroxyl radicals. This leads to a formation of FQ clusters close to the membrane of the digestive vacuole, resulting in the formation of ROS and lipid peroxidation, leading to the death of the parasite. FQ ability to decrease $\mathrm{CD} 4^{+} \mathrm{CD} 25^{+} \mathrm{T}$-cells through rapid action/effect against blood parasites [15] and its ability to maintain the proliferation of spleen cells in the presence of different mitogens [10] have led researchers to suggest FQ as a great alternative to the treatment of $P$. falciparum.

FQ antimalarial activity has become of interest to the researchers and a bridge in finding similar FQ derivatives having antimalarial activities. Some classes of FQ derivatives include hydroxyferroquines, trioxaferroquines, thiosemicarbazone derivatives, ferrocene dual conjugates, chloroquine-bridged ferrocenophanes, and 4-N-substituted derivatives.

3.1. Hydroxyferroquine Derivatives. Three hydroxyferroquine (Figure 5) derivatives were synthesized by Biot et al. [16] to closely mimic hydroxychloroquine. Although derivatives of $F Q$ performed lesser than the original FQ, they were more successful in the inhibition of in vitro growth of $P$. falciparum than CQ. Furthermore, they exhibited antiviral effects in severe acute respiratory syndrome coronavirus [9]. It has also been found that ferroquine and derivatives have antitumor activity. Literature has shown that ferroquine disturbs lysosomal function and therefore inhibits autophagy [17].

\subsection{Trioxaferroquines. Trioxaferroquines (Figure 6) were} reported as potential antimalarial agents against CQ-resistant strains, FcB1 and FcM29 [18]. Trioxaferroquines were shown to have high activity and great ability to clear parasitemia below detectable levels in mice when treated with $10 \mathrm{mg} / \mathrm{kg}$ [18]. Therefore, Trioxaferroquines have been indicated as a new class of antiparasitic agents in vivo and in vitro.

3.3. Thiosemicarbazone Derivatives. Thiosemicarbazones (TSCs) (Figure 7) and FQ analogs display a great activity against different strains of $P$. falciparum. These analogs accumulate in food vacuole and showed a better parasitic activity than enzyme inhibition. The use of TSCs and FQ showed that there may be more than one mode of action. The first is using falcipain-2 as a molecular target and the second is acting independently [16].

3.4. Ferrocene Dual Conjugates. These dual FQ derived models showed a better activity in vitro against NF54 and K1 clones of $P$. falciparum [9]. However, the dual FQ derivative's activity was lower than that of the parent FQ analogs. This could have been the result of oxidative activity in the digestive vacuole [9].

3.5. Chloroquine-Bridged Ferrocenophanes. Salas et al. recorded five disubstituted FQ derivatives [19]. Four of these derivatives were active against different strains of $P$. falciparum especially CQ-resistant $\mathrm{Dd} 2$ and $\mathrm{K} 1$. All of these derivatives were found to interact with the hematin. However, the balance that existed between the lipophilicity and hydrophilicity played a major role in their activities, resulting in the overall success in transportation through the membrane [9]. 


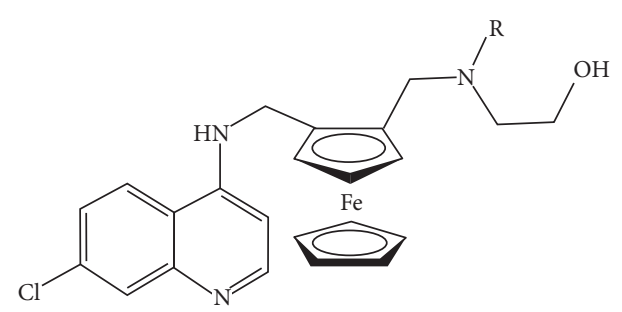

FIGURE 5: Hydroxyferroquine. $\mathrm{R}_{1}, \mathrm{R}_{2}=\mathrm{CH}_{3}$.
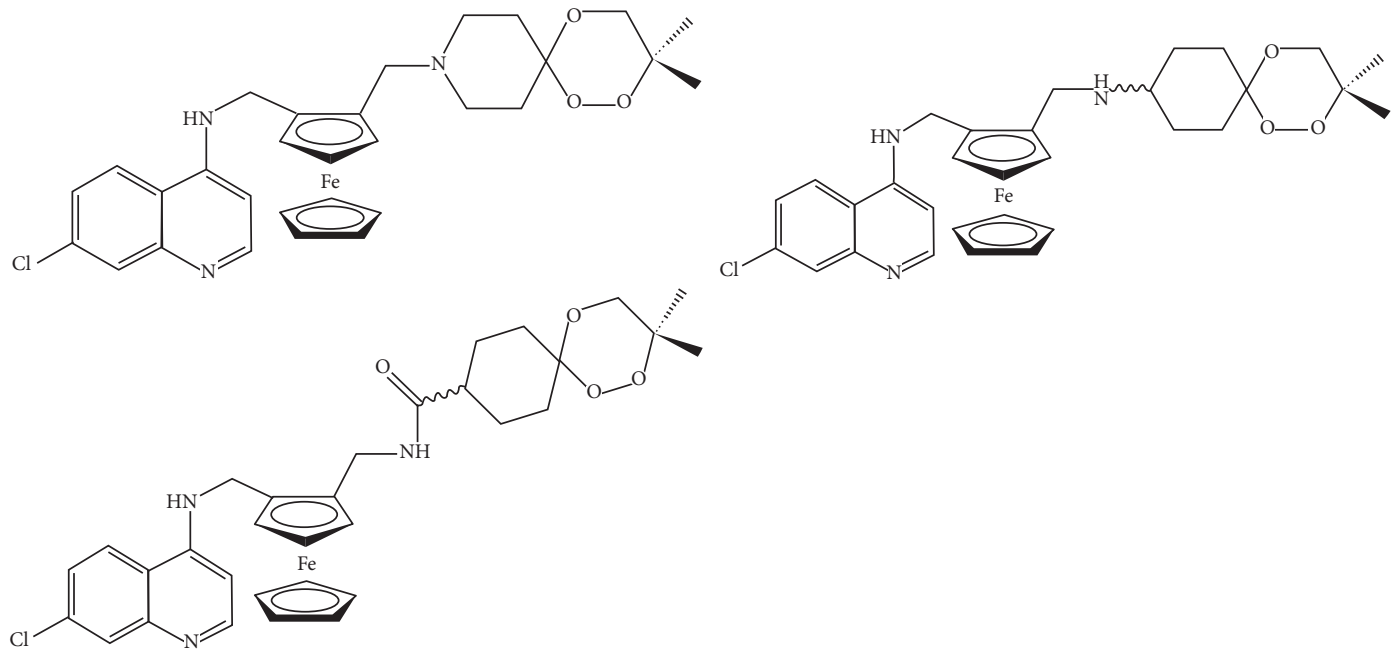

Figure 6: Trioxaferroquine derivatives.<smiles>[R]C([R])=NN([B])C(=S)N([R3])[B]</smiles>

Figure 7: Thiosemicarbazone derivatives.

\section{Mechanism of Action of Organometallic Analogs of Known Antimalarial Drugs and Their Binding Mechanisms}

The inhibition of the formation of hemozoin remains the most acceptable mechanism of action of most antimalarial drugs [5]. The details of the mechanism of action of how these drugs inhibit hemozoin formation remains nebulous [5]. Studies to unravel the mechanism of action of organometallic analogs date back to the early 1990s. Gabay et al. suggested that chloroquine inhibits the release of iron from hemoglobin in the host, thereby depriving the parasite of iron [20] Chloroquine, quinoline methanol, quinine, quinidine, and artemisinin target heme or hematin, and this prevents the formation of hemozoin which is crucial for the survival of the Plasmodium parasite [21, 22]. The aminoquinolines bind with the free hematin and inhibit the crystallization of heme to hemozoin $[5,23]$. Desferrioxamine, an iron chelator, has shown antiplasmodial activity, though it has direct activity against the parasite rather than the host's iron status $[24,25]$. Clinical reports suggest that iron supplementation increases an individual's susceptibility to Plasmodium infection [26-29]. In contrast, other studies have also shown that the prevalence of malaria is not related to iron supplementation [30, 31]. Although, the role of iron in malaria pathogenesis still remains unclear, studies have shown that Plasmodium has a strong affinity to iron and it is essential for its survival. Due to the avidity of Plasmodium to free iron [32], the addition of iron to existing antimalarial molecules has become advantageous in the fight against malaria. This helps to remove the resistance exhibited by Plasmodium to these drugs. Organometallic compounds based on quinoline and other malarial drugs with ferrocene nucleus have been reported to show better activity against Plasmodium spp. clones and strains [33-36]. However, other studies have also reported less active compounds synthesized by incorporating a ferrocenyl moiety into other known antimalarial drugs such as mefloquine and quinine [37] or artemisinin [38].

Ferroquine and its analogs act as resistant reversing agents and are believed to block $P$. falciparum Chloroquine Resistance Transporter (PfCRT), thus maintaining ferroquine in the parasite food vacuole $[39,40]$. The mechanism of action of ferroquine is believed to be similar to that of CQ. However, due to its better lipophilic properties and special conformation, ferroquine is a more potent antimalarial than CQ [41]. Ferroquine is also reported to form complexes with hematin, thereby acting as a more efficacious inhibitor of 
$\beta$-hematin formation than CQ $[33,42]$. Like other quinolines, ferroquine identifies hematin as a major drug target, and the formation of hemozoin is inhibited by their complex $[42,43]$. It has also been suggested that ferrocene only acts as an inhibitor of CQ resistance without enhancing the effectiveness of CQ and has been reported to have no effect when used with CQ without covalent bonding [33]. Furthermore, ferroquine analogs have also been shown to generate hydroxyl radicals which might irreversibly damage the parasite digestive vacuole membrane [14, 42].

The needle-like crystals of hemozoin are bounded by sharp $\{1,0,0\}$ and $\{0,1,0\}$ side faces, which are at right angles to each other, and capped by $\{0,1,1\}$, which is slanted with respect to the needle $\mathrm{c}$-axis, and to a lesser extent by $\{0,0,1\}$ end, and this is perpendicular to the c-axis $[42,44,45]$. An antimalarial drug should bind to face $\{0,0,1\}$ or $\{0,1,1\}$ in order to retard nucleation of hemozoin crystals. However, an effective drug will bind to either the $\{1,0,0\}$ or $\{0,1,0\}$ face [42]. Reports suggest that CQ binds to the $\{0,0,1\}$ and $\{1,0,0\}$ faces [46]. Similar binding to the $\{0,0,1\}$ and $\{1,0,0\}$ faces was also predicted for ferroquine and ruthenocene [42].

Ruthenocene, which is a replacement for the ferrocenyl moiety, has spurred the development of potent antimalarials such as ruthenoquine and its analogs. The antimalarial activity of ruthenoquine has been reported to be similar to that of ferroquine [42, 47, 48]. Unlike ferroquine, ruthenoquine analogs do not generate hydroxyl radicals in the parasite vacuole. They possess weaker binding interaction to hematin as compared to CQ and ferroquine. However, like ferroquine, ruthenoquine has a folded conformation and a high lipophilicity, which enhances the transport of these drugs through the parasite membrane. Also, ruthenoquine demonstrates a stronger parasite vacuolar accumulation [42] as compared to ferroquine.

Trioxaferroquine which is a hybrid composed of artemisinins linked covalently to ferroquine moiety has been suggested to have a synergistic antimalarial effect $[18,49-51]$. The first mode of action is similar to that of ferroquine, which plays a vital role in decreasing the rate of resistance development. The other mode of action of trioxaferroquines is also similar to that of artemisinin. Trioxaferroquines are designed to act as alkylating agents and to easily penetrate within infected erythrocytes [22].

Ferroquine analog conjugated to a gluthatione reductase (GR) inhibitor is another antimalarial prodrug strategy. These compounds seek to cause oxidative stress, mainly by the ferroquine, and to promote the depletion of gluthatione (GSH), an enzyme which protects cells from oxidative damage. GSH is also associated with increased resistance to CQ in P. falciparum [52]. GR inhibitors are usually classified as antimalarial drugs [53]. These ferroquine conjugates with GR inhibitors have been shown to be more potent than the parent GR inhibitors or GHS depletors, though they are less active in comparison to ferroquine [54].

Chloroquine-bridged ferrocenophane derivatives have been reported to overcome CQ resistance [55]. They were also found to be associated with hematin though this association does not play an integral role in determining the in vitro activity. Like ferroquine, CQ-bridged ferrocenophanes possess high lipophilicity which improves their transport through the parasite membranes, hence the high activity of the drugs. Their conformation, compact size, and lipophilicity aid the compounds to escape the mechanisms of resistance by the pathogens [55]. Ferroquine was also reported to accumulate in the parasite vacuole.

\subsection{Comparing Organometallic Antimalarial Compounds and} Their Organic Counterparts. Organometallics have so far proved to be attractive first line antimalarial drug contenders. This is not only due to their high activity against the Plasmodium parasite (which is on an equal footing with that of their organic counterparts); primarily they offer solutions as an alternate class of drugs due to problems of the parasite's resistance [56, 57]. Organometallic complexes have generally been shown to possess very unique physicochemical properties which set them apart from purely organic compounds and have promising applications in drug design $[56,58]$.

Ferroquine is currently the most advanced organometallic antimalarial drug candidate $[11,59]$. The compound is highly active against CQ-susceptible and CQ-resistant $P$. falciparum strains $[8,11,40,42]$. Its mechanism of action has also been shown to be similar to that of CQ, which is the inhibition of hemozoin formation $[8,40]$. However, studies on the antimalarial activity of ferroquine compared to CQ point out certain differentiating factors between the two compounds due to the presence of the ferrocene [42]. For example, ferroquine has much higher lipophilicity (2.95) than CQ (0.85) at $\mathrm{pH}$ of 7.4 and lower $\mathrm{pK}_{\mathrm{a} 1}$ and $\mathrm{pK}_{\mathrm{a} 2}$ values (8.19 and 6.99, respectively) than chloroquine (10.03 and 7.94, respectively) [11].

Ferroquine is more lipophilic than $\mathrm{CQ}$, giving it the ability to permeate cells more efficiently $[11,60]$. This explains why ferroquine is more potent inhibitor of hemozoin formation with an $\mathrm{IC}_{50}$ of 0.8 as compared to CQ which has an $\mathrm{IC}_{50}$ of 1.9 [60]. Ferroquine also inhibits the formation of $\beta$-hematin [11]. Ferroquine is able to produce hydroxyl radicals in the presence of hydrogen peroxide within the acidic environment of the parasite's digestive vacuole [61]. Though the concentration of the hydroxyl radicals is weak, they still are lethal for the parasite and can possibly inhibit reinvasion. CQ on the other hand cannot produce hydroxyl radicals by itself given similar conditions. Thus, not only is ferroquine able to attack the parasite using the hemozoin inhibition mechanism but its antimalarial activity is contributed by other alternate mechanisms which may explain its high activity against CQ-resistant $P$. falciparum strains $[60,61]$.

Ferrocene analogs have been developed for other antimalarial drugs including artemisinin, atovaquone, mefloquine, and quinine $[59,60,62]$. For artemisinin, no enhancement of antimalarial activity has been observed after the introduction of a ferrocene core in its structure [60]. Ferrocene conjugates for atovaquone showed antimalarial activity in the micromolar range after testing it against Toxoplasma gondii and $P$. falciparum while that for mefloquine and quinine has expressed lower activity than 
the parent compounds $[60,63,64]$. Ferrocenyl and ruthenocenyl of mefloquine have however been reported as potential antischistosomal drug candidates [65]. More promising results have been reported for organometallic antimalarial complexes using other metals. Chromium arene-quinolone complexes, iron (II) and ruthenium (II) complexes, and cyrhetrenyl conjugates have also been reported to show antimalarial activity $[48,66-69]$. Chromium arene-CQ complex is more active than CQ against both CQsusceptible and CQ-resistant P. falciparum strains [68].

\section{Plausible Targets for Organometallic Compounds in Antimalarial Drug Discovery}

Inasmuch as success has been achieved with current organometallic antimalarials, it is also imperative to explore them against other relevant Plasmodium drug targets and pathways. Notable among these targets are the digestive vacuole, apicoplast, and the mitochondria. The apicoplast is a plastid within the Plasmodium parasite, which has similarities to plant and algae chloroplasts but lacks the ability to photosynthesize [70]. It is known to arise from endosymbiotic origin and thus plays roles in deoxyribonucleic acid (DNA) replication, transcription, and translation [71].

In other works, the Plasmodium apicoplast has been predicted to be involved in the synthesis of heme, fatty acids, and isoprenoids [72] even though its most biological relevant mechanism is yet to be deciphered [73]. It is important to note that biological processes essential to the parasite are quite unique from that of its mammalian hosts. As such, the apicoplast's potential as an antimalarial drug target has been highly recommended [74]. So far, antimalarial drugs such as ciprofloxacin, rifampicin, and doxycycline have targeted the organelle's DNA replication, transcription, and translation [75]. Antimalarial drug candidates such as fosmidomycin [75] and MMV-08138 [76] have also been shown to target the isoprenoid precursor pathways. An organometallic compound composed of a ferrocenyl substituent in the quinolone ring of ciprofloxacin has been reported to show stronger antimalarial activity than the parent compound [77]. In spite of this, research on developing organometallic compounds that target the Plasmodium apicoplast seems to be inadequate.

The Plasmodium mitochondria is known to be significantly different from that of the human cell and is the source of macromolecules involved in protein synthesis and electron transport process which are potential drug targets [78]. Recently, Basto et al. have reported parasitical activities of dinuclear thiolato-bridged arene ruthenium complexes which were tested against another apicomplexan parasite, $T$. gondii. The complexes caused severe structural modifications in the mitochondrion and were predicted to be capable of exhibiting similar parasitical activities against related apicomplexan parasites [79]. It will be interesting to know the inhibitory activity of the complexes against $P$. falciparum.

The food vacuole is another important target in antimalarial drug design. It houses the hemozoin and contains the aggregation of heme from hemoglobin metabolism. The pathway involves proteases which help in the metabolism of hemoglobin to smaller peptides [78]. Organometallic complexes which target mechanisms within the vacuole such as ferroquine express more potent antiplasmodial activity than CQ. This is due to the presence of the metallic components, giving them unique properties that are lethal within the parasite's vacuolar environment. Recent work has demonstrated that exploration of the food vacuole is already on course, as evidenced by Subramanian et al. when they targeted the Plasmodium parasite by groups of ferrocene derivatives. Their results showed that the gold complex among the organometallic compounds affected the parasite's food vacuole and heme detoxification leading to the death of the parasite [80]. Developing more organometallic antimalarials with more lethal tendencies within the parasite's food vacuole will help in overcoming the drug resistance tendencies of the parasite.

Other new drug targets involved in pathways such as purine biosynthesis, nucleotide synthesis, membrane biosynthesis, and Glycolysis pathways [78] could all be explored for the development of targeted organometallic antimalarial compounds, in order to effectively combat the issues of resistance to antimalarial drugs and to open a new phase in antimalarial drug design.

\section{Conclusion}

Since the emergence of ferroquine, development of organometallic antimalarial compounds has been progressing rapidly. One common way organometallic antimalarials are currently developed is by modifying unwanted properties of a pure organic antimalarial, replacing it with an organometallic moiety. Unique properties such as increased lipophilicity and redox properties induced by the organometallic moiety to the parasite give greater possibilities for being used in antimalarial agents. The organometallic compounds have shown increased potency in relation to their purely organic parent compounds. To promote diversity in the development of antimalarials, organometallics can be applied in targeting other promising drug targets and pathways relevant to the parasite. This new class of malaria therapeutics holds a lot of promise, and soon enough issues of the parasite resistance to drugs may be alleviated.

\section{Conflicts of Interest}

The authors declare that they have no conflicts of interest.

\section{Acknowledgments}

This work was supported by the National Science Foundation (NSF-1818693, NSF-1912104) and the National Institutes of Health (NIH-3U01CA227550-02S1).

\section{References}

[1] S. Kumar, T. R. Bhardwaj, D. N. Prasad, and R. K. Singh, "Drug targets for resistant malaria: historic to future perspectives," Biomedicine \& Pharmacotherapy, vol. 104, pp. 827, 2018. 
[2] A. O. Talisuna, P. Bloland, and U. D'Alessandro, "History, dynamics, and public health importance of malaria parasite resistance," Clinical Microbiology Reviews, vol. 17, no. 1, pp. 235-254, 2004.

[3] D. Dive and C. Biot, "Ferrocene conjugates of chloroquine and other antimalarials: the development of ferroquine, a new antimalarial," ChemMedChem, vol. 3, no. 3, pp. 383-391, 2008.

[4] B. Albada and N. Metzler-Nolte, "Highly potent antibacterial organometallic peptide conjugates," Accounts of Chemical Research, vol. 50, no. 10, pp. 2510-2518, 2017.

[5] M. Navarro, W. Castro, and C. Biot, "Bioorganometallic compounds with antimalarial targets: inhibiting hemozoin formation," Organometallics, vol. 31, no. 16, pp. 5715-5727, 2012.

[6] M. Chinappi, A. Via, P. Marcatili, and A. Tramontano, "On the mechanism of chloroquine resistance in Plasmodium falciparum," PLoS One, vol. 5, no. 11, Article ID e14064, 2010.

[7] X. Nqoro, N. Tobeka, and B. Aderibigbe, "Quinoline-based hybrid compounds with antimalarial activity," Molecules, vol. 22 , no. 12 , p. $2268,2017$.

[8] F. Dubar, J. Khalife, J. Brocard, D. Dive, and C. Biot, "Ferroquine, an ingenious antimalarial drug-thoughts on the mechanism of action," Molecules, vol. 13, no. 11, pp. 2900-2907, 2008.

[9] W. A. Wani, E. Jameel, U. Baig, S. Mumtazuddin, and L. T. Hun, "Ferroquine and its derivatives: new generation of antimalarial agents," European Journal of Medicinal Chemistry, vol. 101, pp. 534-551, 2015.

[10] C. Pierrot, S. Lafitte, D. Dive, L. Fraisse, J. Brocard, and J. Khalife, "Analysis of immune response patterns in naïve and Plasmodium berghei-infected young rats following a ferroquine treatment," International Journal for Parasitology, vol. 35, no. 14, pp. 1601-1610, 2005.

[11] C. Biot, F. Nosten, L. Fraisse, D. Ter-Minassian, J. Khalife, and D. Dive, "The antimalarial ferroquine: from bench to clinic," Parasite, vol. 18, no. 3, pp. 207-214, 2011.

[12] J. S. McCarthy, T. Rückle, E. Djeriou et al., “A phase II pilot trial to evaluate safety and efficacy of ferroquine against early Plasmodium falciparum in an induced blood-stage malaria infection study," Malaria Journal, vol. 15, no. 1, p. 469, 2016.

[13] A. Yayon, Z. I. Cabantchik, and H. Ginsburg, "Susceptibility of human malaria parasites to chloroquine is $\mathrm{pH}$ dependent," Proceedings of the National Academy of Sciences, vol. 82, no. 9, pp. 2784-2788, 1985.

[14] N. Chavain, H. Vezin, D. Dive et al., "Investigation of the redox behavior of ferroquine, a new antimalarial," Molecular Pharmaceutics, vol. 5, no. 5, pp. 710-716, 2008.

[15] T. T. A. Long, S. Nakazawa, S. Onizuka, M. C. Huaman, and H. Kanbara, "Influence of $\mathrm{CD} 4{ }^{+} \mathrm{CD} 25^{+} \mathrm{T}$ cells on Plasmodium berghei NK65 infection in BALB/c mice," International Journal for Parasitology, vol. 33, no. 2, pp. 175-183, 2003.

[16] C. Biot, W. Daher, N. Chavain et al., "Design and synthesis of hydroxyferroquine derivatives with antimalarial and antiviral activities," Journal of Medicinal Chemistry, vol. 49, no. 9, pp. 2845-2849, 2006.

[17] A. Kondratskyi, K. Kondratska, F. V. Abeele et al., "Ferroquine, the next generation antimalarial drug, has antitumor activity," Scientific Reports, vol. 7, no. 1, p. 15896, 2017.

[18] F. Bellot, F. Coslédan, L. Vendier, J. Brocard, B. Meunier, and A. Robert, "Trioxaferroquines as new hybrid antimalarial drugs," Journal of Medicinal Chemistry, vol. 53, no. 10, pp. 4103-4109, 2010.
[19] A. Chipeleme, J. Gut, P. J. Rosenthal, and K. Chibale, "Synthesis and biological evaluation of phenolic Mannich bases of benzaldehyde and (thio)semicarbazone derivatives against the cysteine protease falcipain-2 and a chloroquine resistant strain of Plasmodium falciparum," Bioorganic \& Medicinal Chemistry, vol. 15, no. 1, pp. 273-282, 2007.

[20] T. Gabay, M. Krugliak, G. Shalmiev, and H. Ginsburg, "Inhibition by anti-malarial drugs of haemoglobin denaturation and iron release in acidified red blood cell lysates-a possible mechanism of their anti-malarial effect?" Parasitology, vol. 108, no. 4, pp. 371-381, 1994.

[21] C. H. Kaschula, T. J. Egan, R. Hunter et al., "Structure-activity relationships in 4-aminoquinoline antiplasmodials. The role of the group at the 7-position," Journal of Medicinal Chemistry, vol. 45, no. 16, pp. 3531-3539, 2002.

[22] A. Monti, Y. Coppel, and B. Meunier, "Alkylation of heme by the antimalarial drug artemisinin," Chemical Communications, no. 5, pp. 414-415, 2002.

[23] S. Kumar, R. K. Singh, B. Patial, S. Goyal, and T. R. Bhardwaj, "Recent advances in novel heterocyclic scaffolds for the treatment of drug-resistant malaria," Journal of Enzyme Inhibition and Medicinal Chemistry, vol. 31, no. 2, pp. 173-186, 2016.

[24] V. R. Gordeuk, P. E. Thuma, G. M. Brittenham et al., "Iron chelation as a chemotherapeutic strategy for Falciparum malaria," The American Journal of Tropical Medicine and Hygiene, vol. 48, no. 2, pp. 193-197, 1993.

[25] C. Poltera and T. E. Peto, "Deferoxamine inhibition of malaria is independent of host iron status," The Journal of Experimental Medicine, vol. 168, no. 1, pp. 375-387, 1988.

[26] M. A. Peto, M. M. Goheen, and C. Cerami, "Influence of host iron status on Plasmodium falciparum infection," Frontiers in Pharmacology, no. 5, , 2014.

[27] M. Gwamaka, J. D. Kurtis, B. E. Sorensen et al., "Iron deficiency protects against severe Plasmodium falciparum malaria and death in young children," Clinical Infectious Diseases, vol. 54, no. 8, pp. 1137-1144, 2012.

[28] S. J. Oppenheimer, "Iron and malaria," Parasitology Today, vol. 5 , no. 3, pp. 77-79, 1989.

[29] S. J. Oppenheimer, "Iron and its relation to immunity and infectious disease," The Journal of Nutrition, vol. 131, no. 2, pp. 616S-635S, 2001.

[30] A. Neuberger, J. Okebe, D. Yahav, and M. Paul, "Oral iron supplements for children in malaria-endemic areas," Cochrane Database of Systematic Reviews, 2016.

[31] M. B. van Hensbroek, S. Morris-Jones, S. Meisner et al., "Iron, but not folic acid, combined with effective antimalarial therapy promotes haematological recovery in African children after acute falciparum malaria," Transactions of the Royal Society of Tropical Medicine and Hygiene, vol. 89, no. 6, pp. 672-676, 1995.

[32] T. Greenwood and H. Ginsburg, "Hemoglobin denaturation and iron release in acidified red blood cell lysate-A possible source of iron for intraerythrocytic malaria parasites," Experimental Parasitology, vol. 77, no. 3, pp. 261-272, 1993.

[33] O. Domarle, G. Blampain, H. Agnaniet et al., "In vitro antimalarial activity of a new organometallic analog, ferrocenechloroquine," Antimicrobial Agents and Chemotherapy, vol. 42 , no. 3 , pp. 540-544, 1998.

[34] B. Georges, "Ferrocene-chloroquine analogues as antimalarial agents: in vitro activity of ferrochloroquine against 103 Gabonese isolates of Plasmodium falciparum," Journal of Antimicrobial Chemotherapy, vol. 48, no. 2, pp. 179-184, 2001. 
[35] J. Quirante, F. Dubar, A. González et al., "Ferrocene-indole hybrids for cancer and malaria therapy," Journal of Organometallic Chemistry, vol. 696, no. 5, pp. 1011-1017, 2011.

[36] M. B. P. Pradines, J. F. O. Costa, M. S. De Sá et al., "Antiparasitic and immunomodulatory activities of 1,1-bis(4hydroxyphenyl)- 2-phenyl-but-1-ene and its protected and free 2-ferrocenyl derivative," Drug Development Research, 2010.

[37] C. Biot, L. Delhaes, L. A. Maciejewski et al., "Synthetic ferrocenic mefloquine and quinine analoguesas potential antimalarial agents," European Journal of Medicinal Chemistry, vol. 35, no. 7-8, pp. 707-714, 2000.

[38] L. Delhaes, C. Biot, L. Berry et al., "Novel ferrocenic artemisinin derivatives: synthesis, in vitro antimalarial activity and affinity of binding with ferroprotoporphyrin IX," Bioorganic and Medicinal Chemistry, vol. 8, no. 12, pp. 27392745, 2000.

[39] M. Barends, A. Jaidee, N. Khaohirun, P. Singhasivanon, and F. Nosten, "In vitro activity of ferroquine (SSR 97193) against Plasmodium falciparum isolates from the Thai-Burmese border," Malaria Journal, vol. 6, no. 1, p. 81, 2007.

[40] C. Biot, D. Taramelli, I. Forfar-Bares et al., "Insights into the mechanism of action of ferroquine. Relationship between physicochemical properties and antiplasmodial activity," Molecular Pharmaceutics, vol. 2, no. 3, pp. 185-193, 2005.

[41] Y. C. Olliaro and G. Gasser, "Organometallic compounds in drug discovery: past, present and future," Drug Discovery Today: Technologies, 2019.

[42] F. Dubar, T. J. Egan, B. Pradines et al., "The antimalarial ferroquine: role of the metal and intramolecular hydrogen bond in activity and resistance," ACS Chemical Biology, vol. 6, no. 3, pp. 275-287, 2011.

[43] K. N. Olafson, M. A. Ketchum, J. D. Rimer, and P. G. Vekilov, "Mechanisms of hematin crystallization and inhibition by the antimalarial drug chloroquine," Proceedings of the National Academy of Sciences, vol. 112, no. 16, pp. 4946-4951, 2015.

[44] R. Buller, M. L. Peterson, Ö. Almarsson, and L. Leiserowitz, "Quinoline binding site on malaria pigment crystal: a rational pathway for antimalaria drug design," Crystal Growth and Design, vol. 2, no. 6, pp. 553-562, 2002.

[45] I. Solomonov, M. Osipova, Y. Feldman et al., "Crystal nucleation, growth, and morphology of the synthetic malaria pigment $\beta$-hematin and the effect thereon by quinoline additives: the malaria pigment as a target of various antimalarial drugs," Journal of the American Chemical Society, vol. 129, no. 9, pp. 2615-2627, 2007.

[46] I. Weissbuch and L. Leiserowitz, "Interplay between malaria, crystalline hemozoin formation, and antimalarial drug action and design," Chemical Reviews, vol. 108, no. 11, pp. 4899-4914, 2008.

[47] P. Beagley, M. A. L. Blackie, K. Chibale, C. Clarkson, J. R. Moss, and P. J. Smith, "Synthesis and antimalarial activity in vitro of new ruthenocene-chloroquine analogues," Journal of the Chemical Society, Dalton Transactions, no. 23, pp. 4426-4433, 2002.

[48] N. B. d. Souza, A. C. C. Aguiar, A. C. d. Oliveira et al., "Antiplasmodial activity of iron(II) and ruthenium(II) organometallic complexes against Plasmodium falciparum blood parasites," Memórias Do Instituto Oswaldo Cruz, vol. 110, no. 8, pp. 981-988, 2015.

[49] O. Krettli, F. Benoit-Vical, A. Robert, and B. Meunier, "Preparation and antimalarial activities of "trioxaquines", new modular molecules with a trioxane skeleton linked to a 4- aminoquinoline," ChemBioChem, vol. 1, no. 4, pp. 281-283, 2000.

[50] C. Reiter, T. Fröhlich, M. Zeino et al., "New efficient artemisinin derived agents against human leukemia cells, human cytomegalovirus and Plasmodium falciparum: 2nd generation 1,2,4-trioxane-ferrocene hybrids," European Journal of Medicinal Chemistry, vol. 97, pp. 164-172, 2015.

[51] A. Robert, O. Dechy-Cabaret, J. Cazelles, and B. Meunier, "From mechanistic studies on artemisinin derivatives to new modular antimalarial drugs," Accounts of Chemical Research, vol. 35, no. 3, pp. 167-174, 2002.

[52] H. Atamna and H. Ginsburg, "Heme degradation in the presence of glutathione. A proposed mechanism to account for the high levels of non-heme iron found in the membranes of hemoglobinopathic red blood cells," Journal of Biological Chemistry, vol. 270, no. 42, pp. 24876-24883, 1995.

[53] Z. Ya, E. Hempelmann, and R. H. Schirmer, "Glutathione reductase inhibitors as potential antimalarial drugs. Effects of nitrosoureas on Plasmodium falciparum in vitro," Biochemical Pharmacology, vol. 37, no. 5, pp. 855-860, 1988.

[54] N. Chavain, E. Davioud-Charvet, X. Trivelli et al., "Antimalarial activities of ferroquine conjugates with either glutathione reductase inhibitors or glutathione depletors via a hydrolyzable amide linker," Bioorganic and Medicinal Chemistry, vol. 17, no. 23, pp. 8048-8059, 2009.

[55] P. F. Salas, C. Herrmann, J. F. Cawthray et al., "Structural characteristics of chloroquine-bridged ferrocenophane analogues of ferroquine may obviate malaria drug-resistance mechanisms," Journal of Medicinal Chemistry, vol. 56, no. 4, pp. 1596-1613, 2013.

[56] S. P. Fricker, "Medicinal organometallic chemistry," Platinum Metals Review, vol. 55, no. 4, pp. 229-232, 2011.

[57] C. G. Hartinger and P. J. Dyson, "Bioorganometallic chemistry-from teaching paradigms to medicinal applications," Chemical Society Reviews, vol. 38, no. 2, pp. 391-401, 2009.

[58] G. Gasser and N. Metzler-Nolte, "The potential of organometallic complexes in medicinal chemistry," Current Opinion in Chemical Biology, vol. 16, no. 1-2, pp. 84-91, 2012.

[59] J. Xiao, Z. Sun, F. Kong, and F. Gao, "Current scenario of ferrocene-containing hybrids for antimalarial activity," $E u$ ropean Journal of Medicinal Chemistry, vol. 185, Article ID 111791, 2019.

[60] C. Biot and D. Dive, "Bioorganometallic chemistry and malaria," in Topics in Organometallic Chemistry, pp. 155-193, Springer, Berlin, Germany, 2010.

[61] F. Dubar, C. Slomianny, J. Khalife et al., "The ferroquine antimalarial conundrum: redox activation and reinvasion inhibition," Angewandte Chemie International Edition, vol. 52, no. 30, pp. 7690-7693, 2013.

[62] S. Paitayatat, B. Tarnchompoo, Y. Thebtaranonth, and Y. Yuthavong, "Correlation of antimalarial activity of artemisinin derivatives with binding affinity with ferroprotoporphyrin IX," Journal of Medicinal Chemistry, vol. 40, no. 5, pp. 633-638, 1997.

[63] A. Baramee, A. Coppin, M. Mortuaire, L. Pelinski, S. Tomavo, and J. Brocard, "Synthesis and in vitro activities of ferrocenic aminohydroxynaphthoquinones against Toxoplasma gondii and Plasmodium falciparum," Bioorganic \& Medicinal Chemistry, vol. 14, no. 5, pp. 1294-1302, 2006.

[64] C. Biot, L. Delhaes, L. A. MacIejewski et al., "Synthetic ferrocenic mefloquine and quinine analogues as potential antimalarial agents," European Journal of Medicinal Chemistry, vol. 35, no. 7-8, pp. 707-714, 2000. 
[65] F. d'Orchymont, J. Hess, G. Panic et al., "Synthesis, characterization and biological activity of organometallic derivatives of the antimalarial drug mefloquine as new antischistosomal drug candidates," MedChemComm, vol. 9, no. 11, pp. 19051909, 2018.

[66] R. Arancibia, C. Biot, G. Delaney et al., "Cyrhetrenyl chalcones: synthesis, characterization and antimalarial evaluation," Journal of Organometallic Chemistry, vol. 723, pp. 143-148, 2013.

[67] R. Arancibia, F. Dubar, B. Pradines et al., "Synthesis and antimalarial activities of rhenium bioorganometallics based on the 4-aminoquinoline structure," Bioorganic \& Medicinal Chemistry, vol. 18, no. 22, pp. 8085-8091, 2010.

[68] L. Glans, D. Taylor, C. de Kock et al., "Synthesis, characterization and antimalarial activity of new chromium arene-quinoline half sandwich complexes," Journal of Inorganic Biochemistry, vol. 105, no. 7, pp. 985-990, 2011.

[69] P. Toro, A. H. Klahn, B. Pradines et al., "Organometallic benzimidazoles: synthesis, characterization and antimalarial activity," Inorganic Chemistry Communications, vol. 35, pp. 126-129, 2013.

[70] S. A. Ralph, M. C. D'Ombrain, and G. I. McFadden, "The apicoplast as an antimalarial drug target," Drug Resistance Updates, vol. 4, no. 3, pp. 145-151, 2001.

[71] R. J. M. Wilson, P. W. Denny, P. R. Preiser et al., "Complete gene map of the plastid-like DNA of the malaria parasite Plasmodium falciparum," Journal of Molecular Biology, vol. 261, no. 2, pp. 155-172, 1996.

[72] S. A. Ralph, G. G. van Dooren, R. F. Waller et al., "Tropical infectious diseases: metabolic maps and functions of the Plasmodium falciparum apicoplast," Nature Reviews. Microbiology, vol. 2, no. 3, pp. 203-216, 2004.

[73] L. Lim and G. I. McFadden, "The evolution, metabolism and functions of the apicoplast," Philosophical Transactions of the Royal Society B: Biological Sciences, vol. 365, no. 1541, pp. 749-763, 2010.

[74] G. C. Sadhukhan and A. Mukherjee, "Anti-malarial drug design by targeting apicoplasts: new perspectives," Journal of Pharmacopuncture, vol. 19, no. 1, pp. 7-15, 2016.

[75] C. Y. Botté, F. Dubar, G. I. McFadden, E. Maréchal, and C. Biot, "Plasmodium falciparum apicoplast drugs: targets or off-targets?" Chemical Reviews, vol. 112, no. 3, pp. 1269-1283, 2012.

[76] W. Wu, Z. Herrera, D. Ebert et al., “A chemical rescue screen identifies a Plasmodium falciparum apicoplast inhibitor targeting MEP isoprenoid precursor biosynthesis," Antimicrobial Agents and Chemotherapy, vol. 59, no. 1, pp. 356-364, 2015.

[77] F. Dubar, G. Anquetin, B. Pradines, D. Dive, J. Khalife, and C. Biot, "Enhancement of the antimalarial activity of ciprofloxacin using a double prodrug/bioorganometallic approach," Journal of Medicinal Chemistry, vol. 52, no. 24, pp. 7954-7957, 2009.

[78] C. D. Goodman, H. D. Buchanan, and G. I. McFadden, "Is the mitochondrion a good malaria drug target?" Trends in Parasitology, vol. 33, no. 3, pp. 185-193, 2017.

[79] A. P. Basto, J. Müller, R. Rubbiani et al., "Characterization of the activities of dinuclear thiolato-bridged arene ruthenium complexes against Toxoplasma gondii," Antimicrobial Agents and Chemotherapy, vol. 61, no. 9, 2017.

[80] G. Subramanian, A. Sadeer, K. Mukherjee et al., "Evaluation of ferrocenyl phosphines as potent antimalarials targeting the digestive vacuole function of Plasmodium falciparum," Dalton Transactions, vol. 48, no. 3, pp. 1108-1117, 2019. 